\title{
VHL16 Peptide
}

National Cancer Institute

\section{Source}

National Cancer Institute. VHL16 Peptide. NCI Thesaurus. Code C2740.

A peptide vaccine derived from the von Hippel-Lindau (VHL) tumor suppressor protein, a general transcription factor. VHL16 peptide, also known as S111I, is a point mutation variant of the VHL tumor suppressor protein; the mutation is in amino acid position 111. Vaccination with this agent may stimulate a cytotoxic $\mathrm{T}$-cell response in patients with VHL-associated cancers that express this variant of the VHL tumor suppressor protein. $(\mathrm{NCl} 04)$ 